\title{
A!
}

This is an electronic reprint of the original article.

This reprint may differ from the original in pagination and typographic detail.

Kervinen, Mikael; Välimaa, Alpo; Ramírez-Muñoz, Jhon E.; Sillanpää, Mika

\section{Sideband Control of a Multimode Quantum Bulk Acoustic System}

Published in:

Physical Review Applied

DOI:

10.1103/PhysRevApplied.14.054023

Published: 11/11/2020

Document Version

Publisher's PDF, also known as Version of record

Please cite the original version:

Kervinen, M., Välimaa, A., Ramírez-Muñoz, J. E., \& Sillanpää, M. (2020). Sideband Control of a Multimode Quantum Bulk Acoustic System. Physical Review Applied, 14(5), 1-8. [054023].

https://doi.org/10.1103/PhysRevApplied.14.054023

This material is protected by copyright and other intellectual property rights, and duplication or sale of all or part of any of the repository collections is not permitted, except that material may be duplicated by you for your research use or educational purposes in electronic or print form. You must obtain permission for any other use. Electronic or print copies may not be offered, whether for sale or otherwise to anyone who is not an authorised user. 


\title{
Sideband Control of a Multimode Quantum Bulk Acoustic System
}

\author{
Mikael Kervinen, ${ }^{1}$ Alpo Välimaa, ${ }^{1}$ Jhon E. Ramírez-Muñoz@,${ }^{2}$ and Mika A. Sillanpää ${ }^{1, *}$ \\ ${ }^{1}$ Department of Applied Physics, Aalto University, P.O. Box 15100, FI-00076 Aalto, Finland \\ ${ }^{2}$ Departamento de Física, Universidad Nacional de Colombia, 111321 Bogotá, Colombia
}

(Received 9 June 2020; revised 8 October 2020; accepted 9 October 2020; published 11 November 2020)

\begin{abstract}
Multimode bulk acoustic systems show promise for use in superconducting quantum computation. They can serve as a medium-term memory storage, with exceptional coherence times being demonstrated, and they exhibit a mode density that is physically highly compact. Here, we experimentally demonstrate accessing individual acoustic modes without being hindered by the uniform frequency spacing of the modes. We use sideband control where a low-frequency modulation is applied to the transmon qubit energy. The amplitude of the modulation defines the qubit-acoustic mode coupling and its frequency detunes the acoustic sidebands, therefore selectively enabling the switching on or off of the interaction, and it allows for full control of the individual modes.
\end{abstract}

DOI: 10.1103/PhysRevApplied.14.054023

\section{INTRODUCTION}

Superconducting qubits are among the leading platforms for quantum computing. Besides the qubits themselves, quantum computation needs, at the very least, memory elements and interconnects. For that purpose, quantum hybrid systems $[1,2]$ that combine superconducting qubits with disparate degrees of freedom have been actively investigated. The experimental work includes, e.g., systems containing spin or magnetic degrees of freedom $[3,4]$. A particularly actively explored medium for qubit hybrids consists of mechanical oscillators or acoustic modes in the solid state [5-14]. A harmonic mode is a good candidate for a memory and can also be used for continuous-variable quantum information [15]. Acoustic memory modes can be made to be very long lived, as acoustic resonators with exceptionally long coherence times, far beyond those of superconducting qubits, have been demonstrated [16].

To maximize the benefit of integrating qubits with acoustic modes, the latter should have a compact form factor to be able to support multiple modes on chip, in addition to the long coherence times. High-overtone bulk acoustic wave resonators (HBARs) [17-20] can fulfill both of these criteria. They are naturally multimode systems and they can have exceptional quality factors. These systems can thus reduce the number of required qubits and control lines in order to create more hardware efficient systems [21].

In this work, we discuss and implement an approach to manipulate a qubit-HBAR system by controlling the qubit with longitudinal fields. The method has proven

*Mika.Sillanpaa@aalto.fi to be a useful tool to control superconducting quantum systems. The experimental demonstrations include sideband transitions [22], two-qubit operations [23,24], and a tunable coupler [25], all realized with flux-induced parametric modulation. The sideband transitions have also been used to generate a controllable interaction between a superconducting qubit and multiple microwave resonators [26]. Other experiments have used parametric modulation to generate photon-assisted Landau-Zener interference $[20,27]$ or motional averaging [28]. Here, we show that the longitudinal modulation can be used to provide selective access to the different acoustic modes in a harmonic multimode quantum acoustic system, where a transmon qubit is coupled to a HBAR device. The advantage of the longitudinal field control is that the multimode system can be controlled with pure sinusoidal signals that can be delivered to the qubit more accurately than short flux pulses.

\section{INTERACTION USING LONGITUDINAL MODULATION}

Let us treat a qubit with the transition frequency $\omega_{0}$ between the ground state and the first excited state. The qubit is described by the standard operators $\sigma_{z}, \sigma_{+}$and $\sigma_{-}$. The qubit is coupled to an array of harmonic oscillators with frequencies $\omega_{m}^{(i)}$ for mode $i$, at the coupling energy $g_{m}$, which is supposed to be the same for all of the oscillators. The oscillators are evenly spaced in frequency and the separation is given by the free spectral range (FSR) $\omega_{\mathrm{FSR}}$. The oscillators are described by the annihilation (creation) phonon operators $a_{i}\left(a_{i}^{\dagger}\right)$ for mode $i$. 
The qubit transition frequency is modulated longitudinally by a classical field $H_{z}(t)=(A / 2) \cos \left(\omega_{\bmod } t\right) \sigma_{z}$. Here, $A$ is the amplitude of the modulation and $\omega_{\bmod } \ll \omega_{0}$ is the modulation frequency. The full system under the frequency modulation is described by the Hamiltonian [20]

$$
\begin{aligned}
H^{(n, k)}= & \frac{\left(n \omega_{\mathrm{mod}}-\omega_{0}\right)}{2} \sigma_{z}+\sum_{i}\left(\omega_{i}+k \omega_{\bmod }\right) a_{i}^{\dagger} a_{i} \\
& +\sum_{i} g_{m} J_{n-k}\left(\frac{A}{\omega_{\mathrm{mod}}}\right)\left(\sigma_{+} a_{i}+\sigma_{-} a_{i}^{\dagger}\right),
\end{aligned}
$$

which describes an $n$ th-order qubit sideband interacting at the rate $g_{m} J_{n-k}\left(A / \omega_{\text {mod }}\right)$ with a $k$ th-order acoustic sideband. The modulation generates acoustic and qubit sidebands $(n, k= \pm 1,2, \ldots)$, creating a grid of interweaving modes. In our analysis, we consider only the qubit main band $n=0$. Then, of relevance are the acoustic sidebands that are illustrated in Fig. 1. By selecting the operation point of the qubit suitably, different processes connecting the qubit and the acoustic modes can be realized via the frequency modulation. In Fig. 1(b), the useful operating points are labeled (I)-(III). At point (I), without modulation, the qubit is decoupled from the acoustic modes and single-qubit gates can be realized on the bare qubit. Similarly, point (III), also without modulation, results in the normal resonant qubit-oscillator interaction. In order to access individual acoustic modes through the frequency modulation, the qubit is tuned to point (II), which lies off

(a)

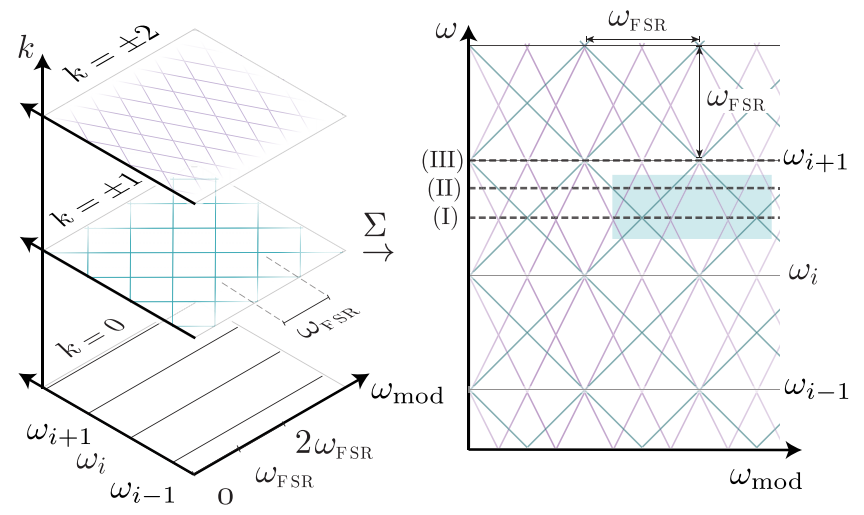

FIG. 1. A graphical illustration of the acoustic sidebands. (a) A stacked representation of the acoustic sidebands from Eq. (1) as given by $\omega_{i}+k \omega_{\text {mod }}$. Each acoustic mode is subject to sidebands (shown up to second order) that depend linearly on the modulation frequency. (b) Acoustic grids superimposed to represent a resonance landscape that we can probe with the qubit. The operating points (I)-(III) are defined with respect to the acoustic modes. The shaded area illustrates the measured parameter range in Fig. 4(a). The spacing between two acoustic modes is given by $\omega_{\mathrm{FSR}}$. the midpoint between consecutive acoustic mode frequencies. At this point, the sidebands become well separated in frequency and they can be accessed individually. At point (I), exactly halfway between two acoustic modes, one can bring the qubit on resonance with two acoustic modes via the modulation.

\section{QUANTUM BULK ACOUSTIC DEVICE}

The experiments are realized with the sample depicted in Fig. 2(a). Compared to our previous device [20], we continue to improve the system parameters by redesigning the device geometry and the choice of materials. The device is implemented in a flip-chip design, where the high-overtone bulk acoustic resonator (HBAR) is overlain on a superconducting Xmon-type transmon qubit [29] fabricated on a silicon substrate [Fig. 2(b)]. The qubit is capacitively coupled to the acoustic modes and the electroacoustic transduction is provided by a $900-\mathrm{nm}$-thick polycrystalline aluminum nitride (AlN) and significantly enhanced by an underlying 60-nm-thick molybdenum (Mo) layer. The HBAR substrate, being $t_{s}=150 \mu \mathrm{m}$-thick single-crystalline sapphire $\left(\mathrm{Al}_{2} \mathrm{O}_{3}\right)$, results in a free spectral range $\omega_{\mathrm{FSR}} / 2 \pi=$ $v_{s} / 2 t_{s} \approx 39 \mathrm{MHz}$ of the overtone modes, where $v_{s}$ is the acoustic velocity in the substrate. A thin AlN layer $(30 \mathrm{~nm})$ resides between the sapphire substrate and molybdenum to match the crystal lattices. The thin film stack is manufactured by OEM Group. The thickness of the film stack ensures that the resonant conditions favor electroacoustic coupling around $5.5 \mathrm{GHz}$.
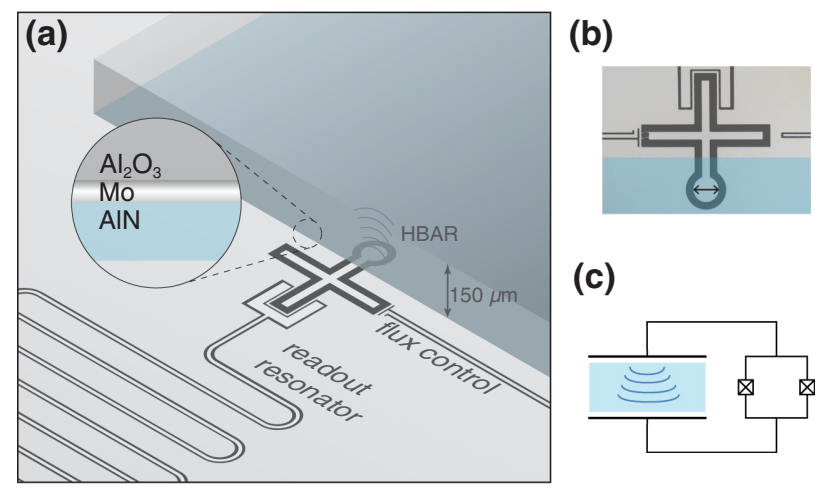

FIG. 2. (a) A schematic of the sample, illustrating the flip-chip assembly of a high-overtone bulk acoustic wave resonator (blue) on top of the Xmon qubit. The acoustic medium is a sapphire crystal that is first covered by a thin layer of molybdenum (Mo, $60 \mathrm{~nm}$ ), on top of which there is an approximately 1- $\mu \mathrm{m}$-thick layer of polycrystalline aluminum nitride. (b) The Xmon-qubit design has separate lines for flux control and transverse drive, a readout resonator with a coupling of $47 \mathrm{MHz}$, and a circular pad for electroacoustic coupling, with a diameter of $72 \mu \mathrm{m}$. (c) A schematic of the transmon-HBAR coupling. The piezoelectric layer acts as a transducer between the electric field of the qubit and the acoustic modes. 
The (super)conducting Mo layer encloses the electrical fields above the Xmon coupling pad in a smaller volume, to provide stronger electric fields that actuate the acoustic modes. In addition, the electric field is likely to be very uniform, which reduces excess acoustic radiation into the bulk and onto the surface. In order to limit the dielectric dissipation in the potentially lossy piezoelectric layer and to mitigate the losses in the qubit [30], the piezoelectric chip only partially covers the qubit. Therefore, the electric field participation ratio of the qubit with the piezoelectric transduction is reduced. However, due to the enhanced coupling provided by the bottom electrode, this approach still retains a strong coupling, while maintaining a good qubit coherence.

The HBAR chip is attached with a two-component epoxy and the chip lies over the qubit without any spacers. We have observed that the natural gap between the two chips due to chip imperfections or small particles that land on the surfaces is on the order of a few hundred nanometers. In the current device, the piezoelectric layer is not shaped in any way, which limits the lateral confinement of the acoustic modes that could result a lower acoustic quality factor, as we have observed in these devices. Another contributing loss factor is the polycrystalline form of the AlN, which may degrade the otherwise excellent lifetimes in the bulk acoustic resonator. Promisingly, recent experimental advances allow for building completely epitaxial bulk acoustic wave resonators with a metallic bottom electrode, creating highly coherent phonon modes [31].

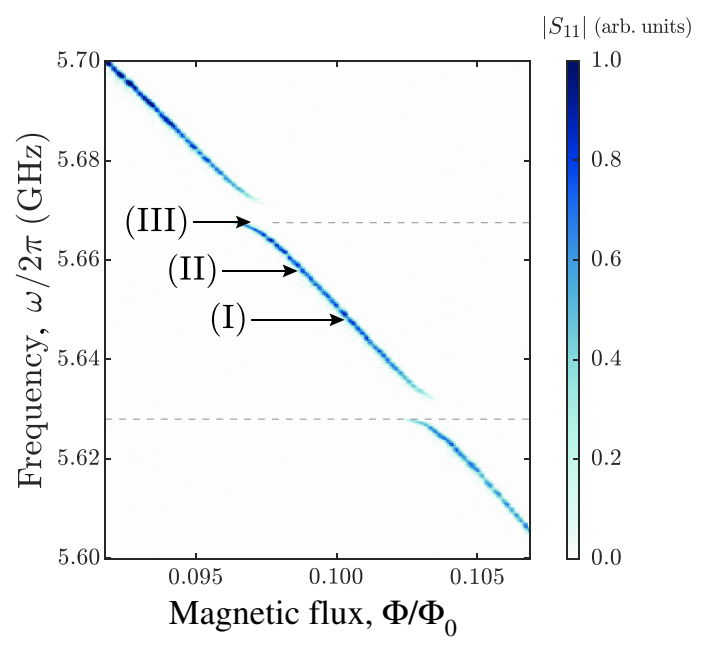

FIG. 3. Two-tone spectroscopy as a function of the flux bias. The operating points (I)-(III) correspond to those in Fig. 1. A dc-flux voltage may be applied to (I) decouple the qubit from the mechanics (without modulation), (III) bring the qubit on resonance with a single nondetuned acoustic mode (without modulation), or (II) access individual acoustic sidebands (with modulation). Additionally, (I) with modulation is used to bring two acoustic modes simultaneously on resonance with the qubit.
The Xmon qubit has symmetric junctions and a maximum frequency of $5.97 \mathrm{GHz}$. However, we operate the qubit away from the sweet spot and detune toward the enhanced qubit-mechanics coupling at $5.65 \mathrm{GHz}$, where the slope of the qubit energy curve is close to linear. The qubit is characterized at the operating point (I) (see Fig. 3), where the qubit is well decoupled from the acoustic modes owing to the detuning being much larger than the coupling. There, we observe the qubit linewidth $\gamma / 2 \pi=0.78 \mathrm{MHz}$ and measure its energy relaxation time $T_{1}=1.09 \mu \mathrm{s}$. By comparing to similar qubit samples without the flip chip, we find that the presence of the piezo does not cause broadening of the qubit linewidth. Therefore, the piezoelectric film is not the current limiting factor of the qubit decoherence. We measure the acoustic lifetime by first exciting the qubit and then swapping the excitation to the mechanical mode. After a variable time delay, the excitation is swapped back to the qubit and its population is measured. We find that the acoustic mode has a lifetime of $T_{1 m}=210$ ns, corresponding to a decay rate of $\gamma_{m} / 2 \pi=0.76 \mathrm{MHz}$, and a phonon quality factor of $Q \approx 7500$. With the coupling rate of $g_{m} / 2 \pi \approx 3.1 \mathrm{MHz}$, the system is in the strong-coupling regime.

\section{SIDEBAND CONTROL OF THE HYBRID SYSTEM}

We study the sideband interaction first in spectroscopic measurements. In Fig. 4(a), the qubit is biased near operation point (II), where individual acoustic sidebands are accessible. As the modulation frequency is varied from 80 to $140 \mathrm{MHz}$, when the resonant condition $\omega_{0}-\omega_{i} \pm \omega_{\bmod }=0$ is fulfilled, we observe the different acoustic sidebands crossing the qubit frequency, at around $\omega_{\bmod } / 2 \pi \simeq 91,105,130 \mathrm{MHz}$. The strength of each anticrossing is given by $2 g_{\text {eff }}=2 g_{m} J_{1}\left(A / \omega_{\text {mod }}\right)$, where $A / 2 \pi=72 \mathrm{MHz}$. These correspond to coupling values of $g_{\text {eff }} / 2 \pi \simeq 1.1,0.99,0.81 \mathrm{MHz}$, respectively.

Next, we study these transitions in a time-resolved manner. We operate the qubit at its resonance frequency. In the pulse sequence in Fig. 4(d), the transmon is first excited with a $\pi$ pulse. We then apply longitudinal flux modulation with a varying length and frequency. After the flux-modulation pulse, the state of the transmon is measured with a measurement pulse. In Fig. 4(b), we display the resulting data as a color map showing the population of the qubit excited state. The horizontal axis is chosen to match that of Fig. 4(a). If the modulation frequency does not coincide with the difference between the qubit and an acoustic mode, we observe a relaxation decay of the bare qubit. Once the modulation frequency corresponds to the resonant condition with an acoustic mode, we observe coherent oscillations between the transmon and the given acoustic mode. These are photon-assisted vacuum Rabi oscillations, where the oscillation rate is controlled by the 

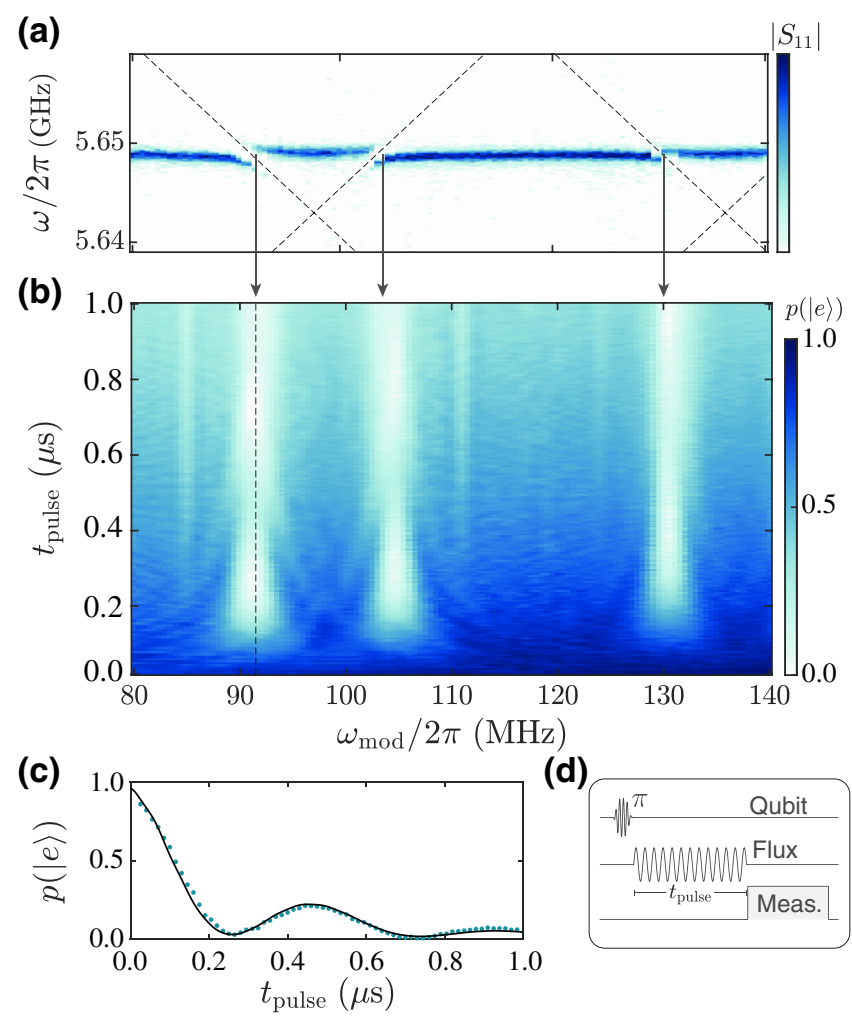

FIG. 4. (a) A frequency-domain measurement to illustrate the operational point and the correspondence to the time-domain measurement and the related mechanical sidebands $(k= \pm 1$, dashed lines). (b) Time-domain spectroscopy. (c) A slice of the time-domain spectroscopy at the modulation frequency $\omega_{\bmod } / 2 \pi=91 \mathrm{MHz}$ [indicated in (b)] together with theoretical modeling overlaid (solid line). (d) A schematic of the pulse sequence, where the qubit is first excited ( $\pi$ pulse), followed by a modulation pulse and readout of the final qubit state. The length of the flux pulse is given by $t_{\text {pulse }}$.

amplitude of the modulation field according to the value of $g_{\text {eff }}$ under the given modulation condition. This allows coherent swapping of the qubit state with the state of a single acoustic mode. As seen in Fig. 4(b), the photonassisted vacuum Rabi oscillations of different acoustic modes are clearly separated, allowing us to selectively access individual modes.

When the transmon population reaches its ground state, the excitation is transferred to the acoustic mode. This is illustrated in detail in Fig. 4(c), which displays a single time trace. Using parametric modulation, the rate of the energy exchange is given by $2 g_{m} J_{1}\left(A / \omega_{\text {mod }}\right)$. Here, the duration of the swap operation is about $240 \mathrm{~ns}$. The fastest energy exchange that one can realize with the sidebands is $2 g_{m} J_{1}(1.84)=0.58 \times 2 g_{m}$, which, with the current experimental value, would be $g_{\text {eff }} / 2 \pi=1.77 \mathrm{MHz}$. However, at this point the coupling to the second-order acoustic sidebands $k= \pm 2$ is also increased, which could induce unwanted spurious interactions. These are faintly visible in Fig. 4 (b) at $\omega_{\text {mod }} / 2 \pi \simeq 85,111,123 \mathrm{MHz}$. By a proper choice of the modulation amplitude and frequency, the coupling to the higher-order sidebands can be effectively minimized [see the Appendix]. The residual contribution can be estimated from $g_{\text {eff }}^{2} /\left(g_{\text {eff }}^{2}+\Delta^{2}\right)$. The phonon-transfer probability to an unwanted sideband mode is at worst $0.5 \%$, so the contribution is minimal. The modulation amplitude is selected such that the maximum peak of the sinusoidal modulation does not bring the qubit on resonance with an acoustic mode. However, due to the high mode density of the HBAR, the modulated qubit energy has to cross with the nearest acoustic modes. The consequence can be estimated using the Landau-Zener tunneling probability, which at typical values can be evaluated as $1-P_{\mathrm{LZ}} \approx 0.1 \%$. The amplitude typically spans a few modes and a time-domain operation includes, for example, a few tens of modulation cycles. When combined, these yield a low-percentage loss of fidelity, which can be further optimized.

If the transmon is biased at operation point (I) such that it is exactly in between two acoustic modes, the modulation creates acoustic sidebands with two modes of opposite $k= \pm 1$. This can be used to couple the transmon to two acoustic modes simultaneously. The two acoustic modes are not necessarily the two closest modes but they can originate from several FSRs away, depending on the modulation frequency. When the qubit starts in its excited state and the two acoustic modes are brought into resonance by the modulation, the qubit excitation is split into the two acoustic modes, creating entanglement of the tripartite system. We show the qubit population in Fig. 5, at several detunings of the modulation frequency. The two acoustic
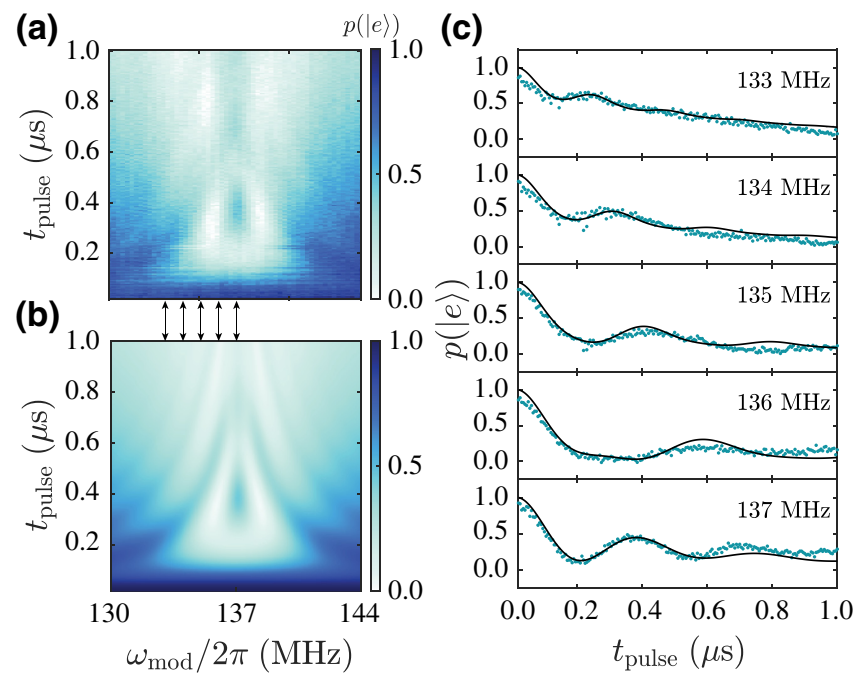

FIG. 5. The (a) experimental and (b) theoretical time-domain study of two acoustic sidebands of the first order, $k= \pm 1$, on resonance with the qubit. The qubit is first prepared in the excited state and then allowed to interact with the acoustic modes. (c) Single traces from (a) and (b) at the modulation frequencies, as indicated. 
modes with opposite modulation frequency dependencies cross the qubit at $137 \mathrm{MHz}$, where we observe simultaneous vacuum Rabi oscillations between the qubit and both sideband modes.

Compared to a case of a single resonant sideband, the oscillations experience faster energy exchange according to $2 g_{\text {eff }} \approx \sqrt{N-1} \times 2 g_{m} J_{1}\left(A / \omega_{\text {mod }}\right)$, where $N=3$. When the modulation frequency coincides exactly with the frequency spacing to the two modes, the qubit is in resonance with the two acoustic modes simultaneously, creating equal populations in the acoustic modes.

\section{AMPLITUDE-CONTROLLED SWITCH}

Next, we use the parametric flux modulation to control the resonant coupling between the qubit and an acoustic mode. We show how the flux modulation can be used to

(a)

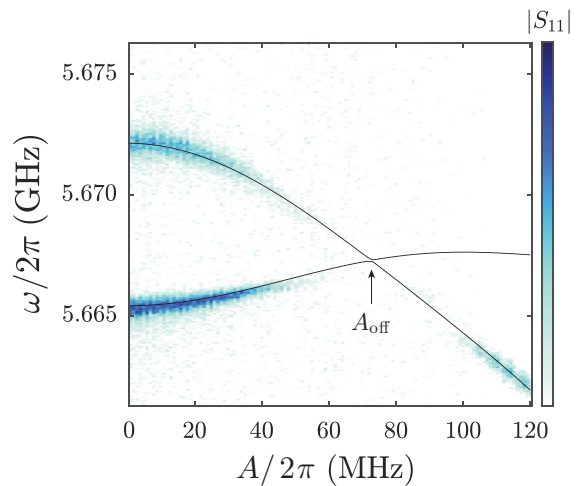

(b)

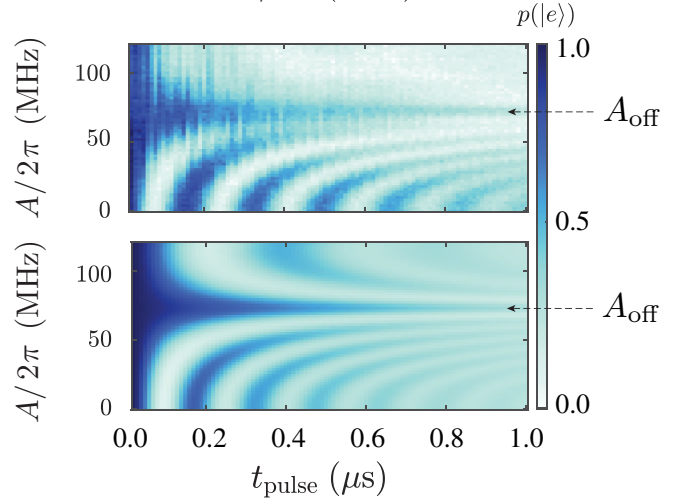

FIG. 6. The control of coupling by the flux-modulation amplitude. (a) The zeroth-order acoustic mode on resonance with the qubit in a spectroscopic measurement and under the flux modulation. The frequency of the modulation is $30 \mathrm{MHz}$ and the amplitude is varied across the root of the Bessel function that modulates the effective qubit-mechanics coupling. (b) Timedomain spectroscopy of the qubit on resonance with an acoustic mode reveals the frequency dependence of the vacuum Rabi oscillations on the flux-modulation amplitude. The coupling, in essence, switches off at $72 \mathrm{MHz}$, where we see the decay of the qubit with no indication of the otherwise coupled acoustic mode. (c) The theoretical modeling of a system comprising qubit and a single acoustic mode on resonance, to reproduce the measurement data in (b). temporarily turn off the acoustic interaction. The use of longitudinal modulation to create a switchable coupling has been previously demonstrated with qubit-qubit and qubit-resonator systems [32].

A spectroscopic measurement of the modulated qubitmechanics system shows an anticrossing in Fig. 6(a). The qubit frequency is fixed on resonance with an acoustic mode and then the modulation is added on top of the static flux bias. As the amplitude is increased, the effective coupling follows the Bessel function $J_{0}\left(A / \omega_{\bmod }\right)$, where the modulation frequency is fixed at $\omega_{\bmod } / 2 \pi=30 \mathrm{MHz}$. Once the modulation-amplitude-to-frequency ratio reaches $A / \omega_{\text {mod }}=2.405$, that is, the root of the zeroth-order Bessel function, the interaction effectively vanishes, demonstrating the possibility of switching off the coupling by selecting the appropriate amplitude-to-frequency ratio. The latter is clearly seen in Figs. 6(b) and 6(c), where the vacuum Rabi oscillations freeze under conditions of zero effective coupling. Due to a slight nonlinearity in the relationship between the dc-flux voltage and the qubit frequency, the driven ac flux induces rectification and contributes to the dc value in proportion with the driving amplitude, thus drifting the qubit away from resonance at higher amplitudes. This is clearly seen in Fig. 6(a), as the bending down of the spectral lines. The theoretical model [solid lines in Fig. 6(a)] matches well with the measurement.

The parametric flux modulation can also be used to control the resonant coupling between the qubit and an

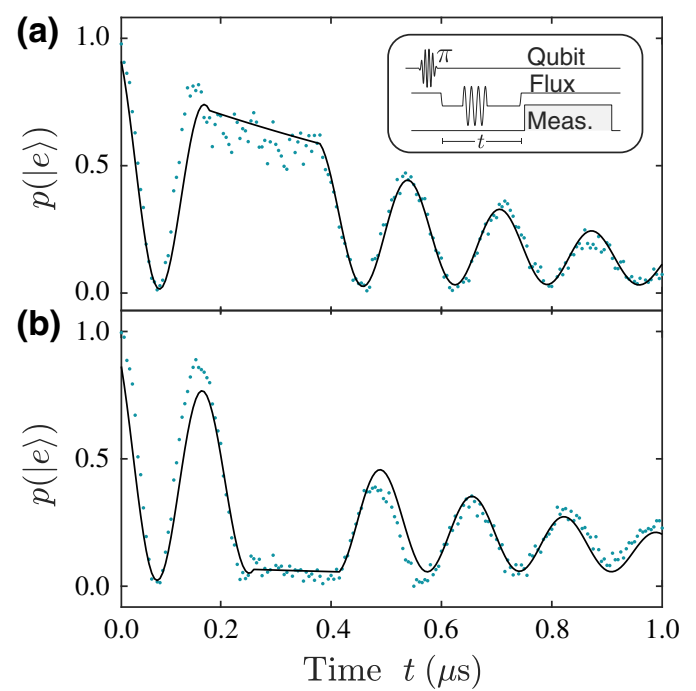

FIG. 7. The flux-modulation-amplitude-controlled switch. The interaction is switched off when the quantum is (a) in the qubit or (b) in the acoustic mode, after which the interaction is recovered. The solid black line is from a master-equation simulation. The inset in (a) shows the pulse sequence, which consists of a $\pi$ pulse to prepare the qubit in the excited state, a dc-flux shift to bring the qubit on resonance with the acoustic mode, flux modulation at an appropriate amplitude and frequency to switch off the interaction, and the qubit readout. 
acoustic mode on the fly during a single time-domain experiment (see Fig. 7). In this case, the modulation pulse is timed as in Fig. 7(a), where the time-dependent modulation parameters yield time-varying qubit-mechanics coupling and interruption of the vacuum Rabi oscillations. When the amplitude of the modulation field is zero, the system experiences regular vacuum Rabi oscillations between the qubit and the acoustic resonator at a rate of $6 \mathrm{MHz}$. The application of the switch is demonstrated in Figs. 7(a) and 7(b). The vacuum Rabi oscillations are allowed to evolve for approximately $200 \mathrm{~ns}$ until a switchoff pulse is applied when the quantum is either in the qubit or in the acoustic mode.

After the switch-off pulse has ended, the system resumes coherent oscillations but with a reduced amplitude due to the energy decay. This shows a proof of concept of the utilization of the acoustic resonator as a memory element, where the quantum state is stored inside the acoustic mode and retrieved back to the qubit.

\section{CONCLUSIONS}

The control techniques that we demonstrate in this work are based on the longitudinal modulation of the qubit energy level, which creates a tunable interaction with the acoustic modes. Although the acoustic modes are equidistant from each other in frequency, by a suitable choice of the parameters one can accurately access individual modes and also bring two acoustic modes on resonance.

We also demonstrate a design for a quantum acoustic system that combines a strong coupling to the acoustic modes with a high qubit coherence. This is achieved by reducing the electric field participation ratio of the qubit with the piezoelectric transduction while enhancing the electromechanical coupling with the usage of a bottom electrode in the HBAR film stack. The device parameters can be greatly improved by combining state-of-theart superconducting qubits with highly coherent acoustic modes.

\section{ACKNOWLEDGMENTS}

This work was supported by the Academy of Finland (Contracts No. 308290, No. 307757, and No. 312057), by the European Research Council (Contract No. 615755), by the Centre for Quantum Engineering at Aalto University, by The Finnish Foundation for Technology Promotion, and by the Wihuri Foundation. We acknowledge funding from the European Union's Horizon 2020 research and innovation program under Grant Agreement No. 732894 (FETPRO HOT). We acknowledge the facilities and technical support of Otaniemi research infrastructure for Micro and Nanotechnologies (OtaNano), which is part of the European Microkelvin Platform. We would like to thank Wayne Crump for useful discussions.

M.K. and A.V. contributed equally to this work.

\section{APPENDIX: EFFECTIVE COUPLING}

In Fig. 1, we display the underlying network of acoustic sidebands. However, the coupling to the sidebands is introduced by the modulation parameters $\left(A, \omega_{\bmod }\right)$. As discussed, by selecting a suitable operating point, one can find regions where the individual acoustic sidebands are accessible. Then, one suppresses the coupling to the higherorder acoustic sidebands via the factor $J_{n-k}\left(A / \omega_{\bmod }\right)$. With a sufficiently small modulation amplitude, or a high enough modulation frequency, the higher-order acoustic sidebands lose a significant coupling to the qubit. This is because the Bessel functions of the first kind $J_{n-k}$ approach zero, when $A / \omega_{\text {mod }}$ approaches zero at an increasing rate with the increasing order of the Bessel functions.

Indeed, one can find that $J_{n-k}\left(A / \omega_{\bmod }\right) \propto\left(A / \omega_{\bmod }\right)^{n-k}$ at small arguments. In Fig. 8(a), we display the Bessel functions for $J_{0}, J_{1}$, and $J_{2}$. At the root of the functions, one can find total suppression of the coupling. The inverse (a)

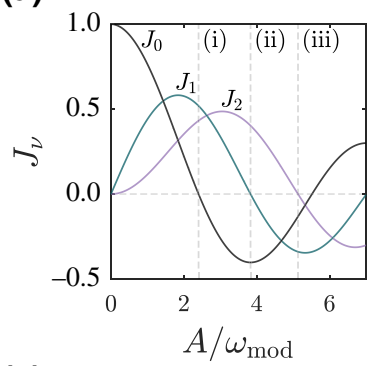

(c)

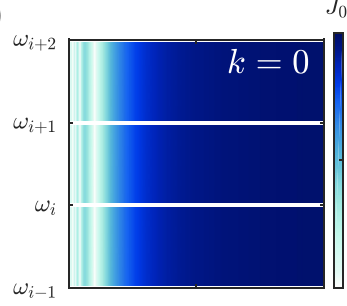

(b)
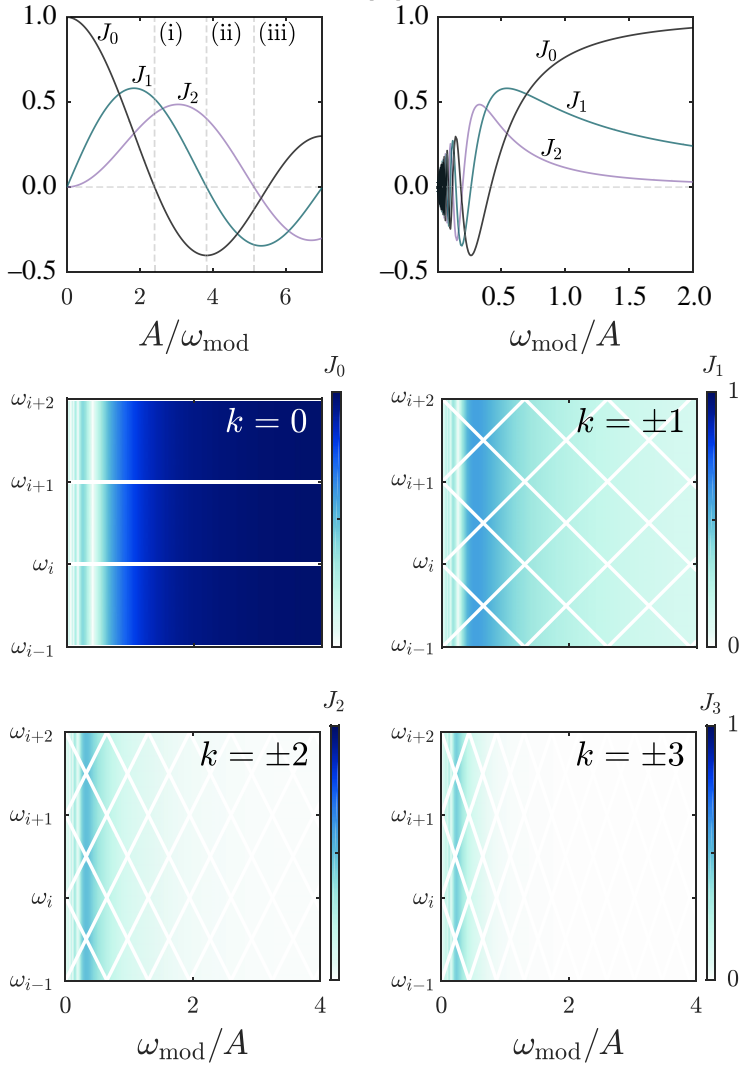

FIG. 8. (a) Bessel functions of the first kind shown for the lowest three orders, $J_{0}, J_{1}$, and $J_{2}$. Positions (i)-(iii) mark the zeros of the functions. (b) With the inverse argument, the functions describe the effective coupling as $\omega_{\bmod }$ is increased. (c) The color map visualizes the value of $g_{\text {eff }}$ for each sideband order $|k|$ and $\omega_{\bmod } / A$ between 0 and 4 . The overlaid white lines illustrate the acoustic sidebands. 
argument $\omega_{\bmod } / A$ that relates to Fig. $4(\mathrm{~b})$ is illustrated in Fig. 8(b). The choice of parameters $\left(A, \omega_{\text {mod }}\right)$ is guided by a sufficiently strong coupling $g_{m} J_{1}$ and a reasonable suppression of higher-order terms. Beyond $\omega_{\bmod } / A>0.5$, the coupling to the higher-order acoustic sidebands is quickly diminished. The sideband energies of the system are mapped to the coupling in Fig. 8(c), with the color map displaying the effective coupling with a fixed $\omega_{\bmod } / \omega_{\mathrm{FSR}}$ ratio.

[1] Z.-L. Xiang, S. Ashhab, J. Q. You, and F. Nori, Hybrid quantum circuits: Superconducting circuits interacting with other quantum systems, Rev. Mod. Phys. 85, 623 (2013).

[2] G. Kurizki, P. Bertet, Y. Kubo, K. Mølmer, D. Petrosyan, P. Rabl, and J. Schmiedmayer, Quantum technologies with hybrid systems, Proc. Natl. Acad. Sci. 112, 3866 (2015).

[3] Y. Kubo, I. Diniz, C. Grezes, T. Umeda, J. Isoya, H. Sumiya, T. Yamamoto, H. Abe, S. Onoda, T. Ohshima, V. Jacques, A. Dréau, J.-F. Roch, A. Auffeves, D. Vion, D. Esteve, and P. Bertet, Electron spin resonance detected by a superconducting qubit, Phys. Rev. B 86, 064514 (2012).

[4] Y. Tabuchi, S. Ishino, A. Noguchi, T. Ishikawa, R. Yamazaki, K. Usami, and Y. Nakamura, Coherent coupling between a ferromagnetic magnon and a superconducting qubit, Science 349, 405 (2015).

[5] M. D. LaHaye, J. Suh, P. M. Echternach, K. C. Schwab, and M. L. Roukes, Nanomechanical measurements of a superconducting qubit, Nature 459, 960 (2009).

[6] A. D. O’Connell, M. Hofheinz, M. Ansmann, R. C. Bialczak, M. Lenander, Erik Lucero, M. Neeley, D. Sank, H. Wang, M. Weides, J. Wenner, J. M. Martinis, and A. N. Cleland, Quantum ground state and single-phonon control of a mechanical resonator, Nature 464, 697 (2010).

[7] J. M. Pirkkalainen, S. U. Cho, J. Li, G. S. Paraoanu, P. J. Hakonen, and M. A. Sillanpää, Hybrid circuit cavity quantum electrodynamics with a micromechanical resonator, Nature 494, 211 (2013).

[8] M. V. Gustafsson, T. Aref, A. F. Kockum, M. K. Ekström, G. Johansson, and P. Delsing, Propagating phonons coupled to an artificial atom, Science 346, 207 (2014).

[9] A. Noguchi, R. Yamazaki, Y. Tabuchi, and Y. Nakamura, Qubit-Assisted Transduction for a Detection of Surface Acoustic Waves Near the Quantum Limit, Phys. Rev. Lett. 119, 180505 (2017).

[10] R. Manenti, A. F. Kockum, A. Patterson, T. Behrle, J. Rahamim, G. Tancredi, F. Nori, and P. J. Leek, Circuit quantum acoustodynamics with surface acoustic waves, Nat. Commun. 8, 975 (2017).

[11] A. N. Bolgar, J. I. Zotova, D. D. Kirichenko, I. S. Besedin, A. V. Semenov, R. S. Shaikhaidarov, and O. V. Astafiev, Quantum Regime of a Two-Dimensional Phonon Cavity, Phys. Rev. Lett. 120, 223603 (2018).

[12] A. Bienfait, K. J. Satzinger, Y. P. Zhong, H.-S. Chang, M.-H. Chou, C. R. Conner, É. Dumur, J. Grebel, G. A. Peairs, R. G. Povey, and A. N. Cleland, Phonon-mediated quantum state transfer and remote qubit entanglement, Science 364, 368 (2019).

[13] P. Arrangoiz-Arriola, E. A. Wollack, Z. Wang, M. Pechal, W. Jiang, T. P. McKenna, J. D. Witmer, R. Van Laer, and A. H. Safavi-Naeini, Resolving the energy levels of a nanomechanical oscillator, Nature 571, 537 (2019).

[14] L. R. Sletten, B. A. Moores, J. J. Viennot, and K. W. Lehnert, Resolving Phonon Fock States in a Multimode Cavity with a Double-Slit Qubit, Phys. Rev. X 9, 021056 (2019).

[15] Z. Leghtas, S. Touzard, I. M. Pop, A. Kou, B. Vlastakis, A. Petrenko, K. M. Sliwa, A. Narla, S. Shankar, M. J. Hatridge, M. Reagor, L. Frunzio, R. J. Schoelkopf, M. Mirrahimi, and M. H. Devoret, Confining the state of light to a quantum manifold by engineered two-photon loss, Science 347, 853 (2015).

[16] G. S. MacCabe, H. Ren, J. Luo, J. D. Cohen, H. Zhou, A. Sipahigil, M. Mirhosseini, and O. Painter, Phononic bandgap nano-acoustic cavity with ultralong phonon lifetime, arXiv:1901.04129.

[17] Y. Chu, P. Kharel, W. H. Renninger, L. D. Burkhart, L. Frunzio, P. T. Rakich, and R. J. Schoelkopf, Quantum acoustics with superconducting qubits, Science 358, 199 (2017).

[18] M. Kervinen, I. Rissanen, and M. Sillanpää, Interfacing planar superconducting qubits with high overtone bulk acoustic phonons, Phys. Rev. B 97, 205443 (2018).

[19] Y. Chu, P. Kharel, T. Yoon, L. Frunzio, P. T. Rakich, and R. J. Schoelkopf, Creation and control of multi-phonon Fock states in a bulk acoustic-wave resonator, Nature 563, 666 (2018).

[20] M. Kervinen, J. E. Ramírez-Muñoz, A. Välimaa, and M. A. Sillanpää, Landau-Zener-Stückelberg Interference in a Multimode Electromechanical System in the Quantum Regime, Phys. Rev. Lett. 123, 240401 (2019).

[21] C. T. Hann, C.-L. Zou, Y. Zhang, Y. Chu, R. J. Schoelkopf, S. M. Girvin, and L. Jiang, Hardware-Efficient Quantum Random Access Memory with Hybrid Quantum Acoustic Systems, Phys. Rev. Lett. 123, 250501 (2019).

[22] J. D. Strand, M. Ware, F. Beaudoin, T. A. Ohki, B. R. Johnson, A. Blais, and B. L. T. Plourde, First-order sideband transitions with flux-driven asymmetric transmon qubits, Phys. Rev. B 87, 220505 (2013).

[23] S. A. Caldwell, N. Didier, C. A. Ryan, E. A. Sete, A. Hudson, P. Karalekas, R. Manenti, M. P. da Silva, R. Sinclair, and E. Acala et al., Parametrically Activated Entangling Gates Using Transmon Qubits, Phys. Rev. Appl. 10, 034050 (2018).

[24] X. Li, Y. Ma, J. Han, T. Chen, Y. Xu, W. Cai, H. Wang, Y. P. Song, Z.-Y. Xue, Z. Yin, and L. Sun, Perfect Quantum State Transfer in a Superconducting Qubit Chain with Parametrically Tunable Couplings, Phys. Rev. Appl. 10, 054009 (2018).

[25] D. C. McKay, S. Filipp, A. Mezzacapo, E. Magesan, J. M. Chow, and J. M. Gambetta, Universal Gate for FixedFrequency Qubits via a Tunable Bus, Phys. Rev. Appl. 6, 064007 (2016).

[26] R. K. Naik, N. Leung, S. Chakram, P. Groszkowski, Y. Lu, N. Earnest, D. C. McKay, J. Koch, and D. I. Schuster, Random access quantum information processors using multimode circuit quantum electrodynamics, Nat. Commun. 8, 1 (2017). 
[27] M. P. Silveri, K. S. Kumar, J. Tuorila, J. Li, A. Vepsäläinen, E. V. Thuneberg, and G. S. Paraoanu, Stückelberg interference in a superconducting qubit under periodic latching modulation, New J. Phys. 17, 043058 (2015).

[28] J. Li, M. P. Silveri, K. S. Kumar, J. M. Pirkkalainen, A. Vepsäläinen, W. C. Chien, J. Tuorila, M. A. Sillanpää, P. J. Hakonen, E. V. Thuneberg, and G. S. Paraoanu, Motional averaging in a superconducting qubit, Nat. Commun. 4, 1420 (2013).

[29] R. Barends, J. Kelly, A. Megrant, D. Sank, E. Jeffrey, Y. Chen, Y. Yin, B. Chiaro, J. Mutus, and C. Neill, et al., Coherent Josephson Qubit Suitable for Scalable Quantum Integrated Circuits, Phys. Rev. Lett. 111, 080502 (2013).
[30] C. Wang, C. Axline, Y. Y. Gao, T. Brecht, Y. Chu, L. Frunzio, M. H. Devoret, and R. J. Schoelkopf, Surface participation and dielectric loss in superconducting qubits, Appl. Phys. Lett. 107, 162601 (2015).

[31] V, J. Gokhale, B. P. Downey, D. S. Katzer, N. Nepal, A. C. Lang, R. M. Stroud, and D. J. Meyer, Epitaxial bulk acoustic wave resonators as highly coherent multi-phonon sources for quantum acoustodynamics, Nat. Commun. 11, 2314 (2020).

[32] Y. Wu, L.-P. Yang, M. Gong, Y. Zheng, H. Deng, Z. Yan, Y. Zhao, K. Huang, A. D. Castellano, W. J. Munro, K. Nemoto, D.-N. Zheng, C. P. Sun, Y. Liu, X. Zhu, and L. Lu, An efficient and compact switch for quantum circuits, npj Quantum Inf. 4, 50 (2018). 